\title{
RESONANCE AND QUENCHING OF THE THIRD PRINCIPAL SERIES LINE OF CAESIUM
}

\author{
By C. Boeckner
}

\section{ABSTRACT}

A helium discharge is used as a source for the study of the resonance radiation from the third principal series line of caesium. This is possible, due to the coincidence of a strong helium line with the caesium line. A number of caesium arc lines are also observed in the fluorescent radiation; they have their origin in lower levels populated by radiation transitions from the initial level $\left(4 P_{1 / 2}\right)$. The effect of helium on the fluorescent radiation is studied. It is found that collisions between helium and caesium atoms in the $4 P_{1 / 2}$ state transfer the latter only to states differing by less than several hundredths of a volt from the initial state. Transfers to states differing by as much as 0.18 volt are improbable.

\section{CONTENTS}

I. Introduction 13

II. Experimental

III. Results

IV. Discussion. 15

\section{INTRODUCTION}

In another paper ${ }^{1}$ from this laboratory it was noted that the strong 3888 helium line coincided with the third principal series line of caesium and that it was markedly absorbed by caesium vapor. This suggested that a helium tube would serve as an excellent source for the study of caesium resonance radiation; the obvious reasons being the simplicity and intensity of the source and absence of selfreversal. The experiment of illuminating caesium vapor with a helium discharge tube was then carried out and it was found that the expected fluorescence could, indeed, be detected. Fifteen-minute exposures with a Hilger $E_{2}$ quartz spectrograph were usually sufficient to photograph the strong lines.

Little work has been done on the excitation of resonance of the higher alkali series lines. Lord Rayleigh ${ }^{2}$ illuminated sodium vapor with the second principal series line of sodium and obtained the $D$ lines in emission; Cario and Franck ${ }^{3}$ later repeated the experiments. Recently C. J. Christensen and G. K. Rollefson ${ }^{4}$ in a similar experiment observed the second line itself and obtained a value for the ratio of the second line to the $D$ lines.

The present paper deals with the fluorescence appearing when caesium atoms are raised to the $4 P_{1 / 2}$ state (the initial state of one component of the third principal series doublet) and the effect of helium in altering the fluorescence.

\footnotetext{
B. S. Jour. Research (in press).

2 R. J. Strutt, Proc. Roy. Soc., London (A), 96, p. 272; 1919.

3 Cario and Franck, Zs. f. Phys., 11, p. 87; 1925.

- C. J. Christensen and G. K. Rollefson, Phys. Rev., 34, p. 1157; 1929.
} 


\section{EXPERIMENTAL}

A simple capillary cold electrode discharge tube containing helium at about $1 \mathrm{~cm}$ pressure was used as a source. The tube was operated at 10,000 volts from a transformer; the primary current being about 8 amperes.

The resonance tube was of the type commonly used in the study of resonance and scattering. It was about $30 \mathrm{~cm}$ long and was drawn out at one end into a light trap. On the other end was sealed a flat glass window. To reduce scattering, a platinum diaphragm with a narrow slit was placed inside of the tube about a centimeter in back of the window, thus shielding it from the direct light of the discharge. The slit was focused on the spectrograph slit. The resonance tube was illuminated by placing the discharge tube near it, but several centimeters away to reduce scattering from the window and light traps. It was not necessary to use great precautions against scattering except for observations on the resonance line itself, since the lines in the source were in no other case near caesium lines.

To maintain the required caesium vapor pressure the discharge tubes and resonance tubes were placed in a furnace and maintained at a temperature of about $200^{\circ} \mathrm{C}$. A small appendix to the resonance tube projected from the furnace and was kept at a somewhat lower temperature. This arrangement insured that the condensed caesium be in the cooler appendix and permitted a definite control of the vapor pressure. The appendix was usually kept at a temperature of $120^{\circ} \mathrm{C}$. (corresponding to a vapor pressure of $0.001 \mathrm{~mm}$ ). This pressure of caesiun was found to give the most intense fluorescence. In some of the work the resonance tube was kept on the pumps to insure a good vacuum.

The helium for the quenching experiments was obtained from tanks supplied by the Navy Department and was purified by passage through a charcoal trap immersed in liquid air.

A Hilger $E_{2}$ quartz spectrograph was used for photographing the fluorescence. For photographing lines in the near infra-red, neocyanin stained plates were used. These were kindly prepared by B. H. Carroll, of the photographic emulsions laboratory.

\section{RESULTS}

A list of the lines appearing in the fluorescent spectrum is given below. Rough estimates of the intensities are also given. These were obtained by calibrating the plate with light from a tungsten filament at $2,900^{\circ}$ and estimating photographic densities visually. Comparisons at different helium pressures were made by using the helium lines in the scattered light as standards:

\begin{tabular}{|c|c|c|}
\hline No foreign gas & $\begin{array}{l}\text { Wave } \\
\text { lengths }\end{array}$ & $4 \mathrm{~mm}$ helium \\
\hline $1 S-4 P_{1 / 2}(10) \ldots$ & $3,888.6$ & $1 S-4 P_{1 / 3}$ \\
\hline $\begin{array}{l}1 S_{-2} P_{3 / 3}(10) \\
2 P_{1 / 2} \\
2 P_{3 / 5}-3 S \text { taint } \\
\text { faint }\end{array}$ & $\begin{array}{l}8,521.4 \\
7,609.7 \\
7,944.1\end{array}$ & $1 S-2 P_{3 / 2}(10)$ \\
\hline $2 P_{1 / 9}-4 D_{3 / 2}$ taint & $\begin{array}{l}8,761.4 \\
6,973.1 \\
6,723.2 \\
4,593.2 \\
4,555.3\end{array}$ & $\begin{array}{l}2 P_{3 / 2}-5 D_{3 / 2,3 / 3}(10) \\
2 P_{1 / 9}-5 D_{3 / 2}(10) \\
1 S-3 P_{1 / 2}(.5) \\
1 S-3 P_{3 / 2}(.5)\end{array}$ \\
\hline
\end{tabular}


B. S. Journal of Research, RP184

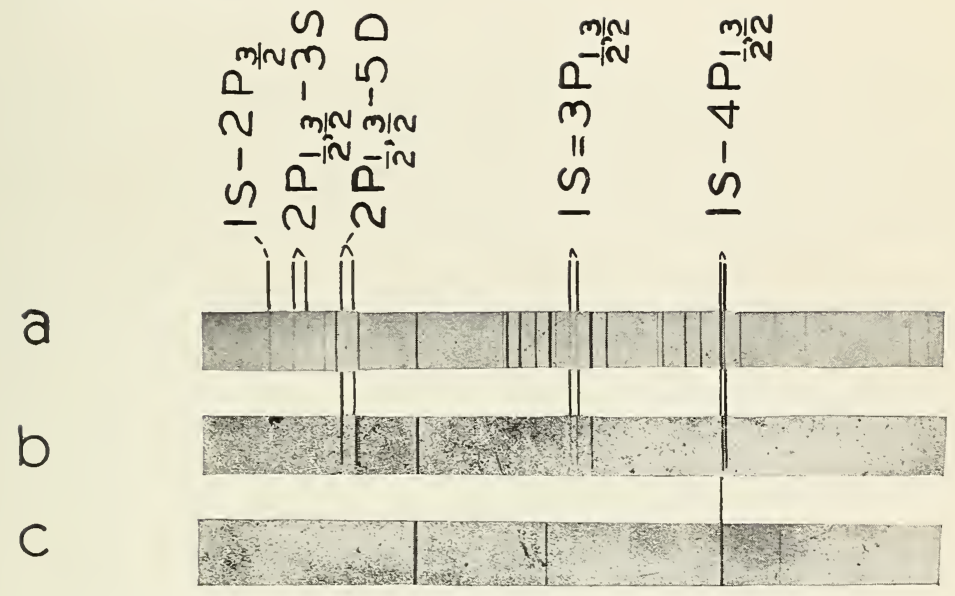

FIgURE 1.-Caesium fluorescence excited by a helium discharge
(a) Photograph showing infra-red lines, some foreign gas.
(b) Fluorescence obtained with $4 \mathrm{~mm}$ helium.
(c) Fluorescence obtained with no foreign gas. 
The most striking effect of the addition of helium is the appearance with great intensity of the doublet $2 P_{1 / 2}-5 D_{3 / 2}, 2 P_{3 / 2}-5 D_{3 / 2,5 / 2}$. The disappearance of the doublet $\left(2 P_{1 / 2}-3 S\right),\left(2 P_{3 / 2}-3 S\right)$ is also interesting.

The principal series doublet $1 S-4 P_{1 / 2}, 3 / 2$ seems to attain its normal intensity ratio at $4 \mathrm{~mm}$ of helium. At zero pressure the intensity of $1 S-4 P_{1 / 2}$ is 10 ; of $1 S-4 P_{3 / 2}$ is 0 ; at $0.6 \mathrm{~mm}$ of helium $1 S-4 P_{1 / 2}$ is 4 , and $1 S-4 P_{3 / 2}$ is 2 ; at $4 \mathrm{~mm} 1 S-4 P_{1 / 2}$ is 2 and $1 S-4 P_{3 / 2}$ is 4 .

The intensity of the first principal series line $1 S-2 P_{3 / 2}$ is not noticeably changed by the addition of helium. The actual magnitude of intensity of this line may be greater than that observed, due to the absorption of the fluorescent radiation in the tube.

The intensity of the fluorescent radiation varies with the caesium pressure and passes through a maximum at about $0.001 \mathrm{~mm}$. Whether the decrease at higher pressures is due to absorption of the exciting light before it reached the field of view, absorption of the fluorescent radiation in the tube, or to actual quenching by caesium, is uncertain.

\section{DISCUSSION}

The reasons for the appearance of the lines observed in fluorescence is best seen by the aid of the level diagram in Figure 2. With no foreign gas in the resonance tube the $4 P_{1 / 2}$ level is strongly populated. The radiation transition to the $1 S$ from this level gives rise to the observed line $1 S-4 P_{1 / 2}$. The transitions from $4 P_{1 / 2}$ to all the lower $S$ and $D$ levels undoubtedly occur, but their wave lengths lie too far in the red to be detected by the photographic method. These lower $S$ and $D$ levels, populated by transitions from the $4 P_{1 / 2}$ level, give rise to some lines that can be detected by the use of a neocyanin stained plate; $2 P_{1 / 2}, 3 / 2-3 S \lambda=7,944,7,609$ are observed as well as $2 P_{1 / 2}-4 D_{3 / 2}$ $\lambda=8,761$. These transitions, as well as the unobserved ones $2 P-2 S$ and $2 P-3 D$, populate the $2 P$ levels and give rise to the strong-line $1 S-2 P_{3 / 2}$. The other component of this doublet, $1 S-2 P_{1 / 2}$ lies too far in the red to be detected by the photographic plate. It is conceivable that the $3 P$ levels could be populated by transitions from $3 S$ and $4 D$, but the second principal series doublet originating from them $\left(1 S-3 P_{1 / 2}, 3 / 2\right)$ could not be observed when a good vacuum existed in the tube. The fact that $1 S-4 P_{1 / 2}$ and $1 S-2 P_{3 / 2}$ have the same order of intensity indicates that the return to $1 S$ from $4 P_{1 / 2}$ directly has the same order of probability as the return by the indirect route by way of $2 P$. This is in agreement with the work of Christensen and Rollefson, ${ }^{5}$ who found that the ratio of intensities of the first principal series line to the second was 1.7 when the second was excited optically.

The results obtained upon the addition of helium are best explained by the assumption that collisions of the second kind in which more than several hundredths of a volt are dissipated as kinetic energy are relatively improbable.

The appearance of the strong $1 S-4 P_{3 / 2}$ and $2 P-5 D$, lines is brought about by the displacement of the atoms from the initial $4 P_{1 / 2}$ level to the neighboring levels $4 P_{3 / 2}$ and $5 D$. The $4 P_{3 / 2}$ level is 0.01 volts and the $5 D$ levels 0.043 volts higher than the initial $4 P_{1 / 2}$ level. The difference in energy must, of course, come from the thermal energy of the colliding atoms. 
The fact that the lines $2 P-3 S$ do not increase but decrease in intensity when helium is added indicates that the transfer of atoms by collision from the $4 P_{1 / 2}$ state to the $3 S$ state, 0.18 volts lower, is improbable. Thus the probability of transfer of energy between states 0.18 volts apart is much less than that between states 0.043 volts apart $\left(2 P_{1 / 2}\right.$ to $\left.5 D\right)$. A rough estimate based on the intensity of the strong $2 P_{1 / 2}-5 D$ and the intensity at $2 P_{3 / 2}-3 S$ that could just be detected puts the ratio of these probabilities as less than $1: 20$.

The disappearance of the $2 P-3 S$ lines can be explained by the decrease in population of the $4 P$ levels by transfer of atoms to the $D$ levels. Since the $3 S$ state is populated by radiation transfers from the $P$ states but not from the $D$ states the population of the $3 S$ state must decrease.

Upon the above idea the faint appearance of the lines $1 S-3 P_{1 / 2,3 / 2}$ upon the addition of helium must be explained by radiation transfer to the $3 P$ levels. It does not seem reasonable to ascribe these lines to collision transfer from the $4 P$ state, since the difference in energy is about 0.5 volt.

These observations seem to be incompatible with the related work of Cario and Franck ${ }^{6}$ upon sodium vapor. They illuminated sodium vapor with the second principal series line and observed that the $D$ lines were greatly increased by the presence of a few millimeters of argon. They also observed that the $D$ light emitted in this case was little absorbed as compared to "oridinary" $D$ light.

These facts were explained by saying that atoms were brought down from the $3 P$ to the $2 P$ state by collisions of the second kind. The difference in energy of 1.6 volts appeared as kinetic energy of translation which meant that the $\mathrm{D}$ lines emitted were broadened by the Doppler effect and, hence, were little absorbed.

The work of Christensen and Rollefson ${ }^{7}$ would make any increase in the $D$ light of more than 60 per cent impossible. They state that 63 per cent of the atoms in the $3 P$ state return to $1 S$ by way of the $2 P$ state; the most any collision process could do would be to bring about the return of the other 37 per cent (that ordinarily returns directly to $1 S$ by radiation) by the same process.

The small probability of collisions of the second kind with dissipation of 0.2 volt as kinetic energy is not surprising. The quenching of the 2,537 resonance radiation of mercury vapor by helium consists in the transfer of the mercury from the $2 P_{1}$ to the metastable $2 P_{0}$ state, 0.22 volts lower. It is found that $1 \mathrm{~cm}$ of helium quenches to the extent of 5 per cent, indicating a small probability for the process.

In the experiments of Beutler and Jospehi ${ }^{8}$ it is also found that interchanges of energy between excited mercury and sodium, involving an appearance of more than 0.15 volts as kinetic energy, are relatively improbable.

Some figures for the efficiency of several quenching reactions are as follows: Transfer of the sodium atom from $2 P_{1 / 2}$ to the $2 P_{3 / 2}$ state ${ }^{9}$ by a helium neon mixture and argon $(\Delta V=0.002$ volts), here every kinetic theory collision is effective; quenching of $\mathrm{Hg}$ resonance ${ }^{10}$ by argon and helium $(\Delta V=0.22$ volts), for argon 0.05 and for helium

- See footnote 3, p. 13.

7 See footnote 4, p. 13.

H. Kallman u. F., London, Zeit. f. Phys. Chem., B 2, p. 207; 1929.

W. Lochte Holtgreven, Zeit. 1 . Phys., 47, p. 369; 1928.

10 E. Gaviola, Phys, Rev., 34, p. 1049; 1929. 
0.005 of the kinetic theory collisions are effective; quenching of the $D$ light resonance of sodium ${ }^{11}$ by a helium neon mixture $(\Delta V=2.1$ volts); here 0.017 of the kinetic theory collisions are effective.

It is not possible to give a precise figure for the collision radius for the transfer of caesium atoms in the $4 P_{1 / 2}$ to the $4 P_{3 / 2}$ and $5 D$ states, since the life time of the $4 P_{1 / 2}$ state is unknown. The half life of the

\section{volts}

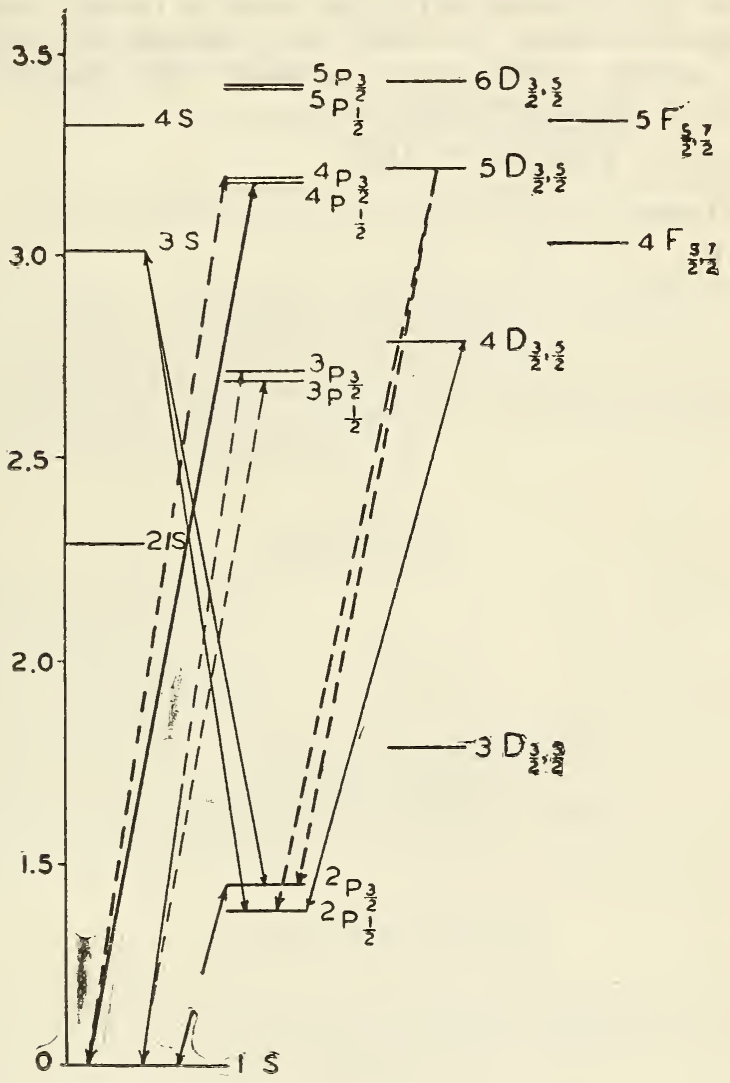

Figure 2.-Level diagram of caesium

Full lines represent transitions observed with no foreign gas, dotted lines transitions observed with helium present.

caesium $2 P$ states is probably the same as that of sodium, ${ }^{12} 3.7 \times 10^{-8}$ seconds.

Canal ray experiments show that the higher states of a series have a longer life than the first state, but rarely longer than $10^{-7}$ seconds. ${ }^{13}$ Taking $10^{-7}$ as a fair value for the life of the $4 P_{1 / 2}$ state and $1 \mathrm{~mm}$ of helium as the pressure at which a collision or a radiation transfer is

13 Hirsch and Döpel, Ann. d. Phys., 17, p. 963; 1929. 
equally probable, one obtains $3.1 \times 10^{-8} \mathrm{~cm}$ for the sum of the helium and caesium radii. The kinetic theory value for helium and normal caesium is $2.9 \times 10^{-8} \mathrm{~cm}$. Thus approximately every kinetic theory collision is effective.

It is interesting to note that Mohler and Boeckner ${ }^{14}$ obtain very great radii for the quenching of the higher excited caesium states by caesium itself. For example, normal caesium apparently reacts with caesium in the $4 P$ state with a radius which is 10 times the kinetic theory value for normal atoms. At $0.001 \mathrm{~m}$ pressure, 0.1 per cent of atoms in the $4 P$ state and 20 per cent in the $6 P$ state react with neutral caesium atoms to form ions instead of radiating. This quenching action would render difficult the study of resonance radiation from the higher series lines.

Washington, February 8, 1930.

is See footnote 1, p. 13. 